\title{
Influence of the deGennes extrapolation parameter on the resistive state of a superconducting strip
}

\author{
J. Barba-Ortega ${ }^{\mathrm{a}, *}$, Edson Sardella $^{\mathrm{b}}$, R. Zadorosny ${ }^{\mathrm{c}}$ \\ a Grupo de Física Mesoscópica, Departamento de Física, Universidad Nacional de Colombia, Bogotá, Colombia \\ ${ }^{\mathrm{b}}$ Departamento de Física, Universidade Estadual Paulista (UNESP), Faculdade de Ciências, Caixa Postal 473, 17033-360, Bauru-SP, Brazil \\ c Departamento de Física e Química, Universidade Estadual Paulista (UNESP), Faculdade de Engenharia de Ilha Solteira, Caixa Postal 31, 15385-000 Ilha \\ Solteira-SP, Brazil
}

\section{A R T I C L E I N F O}

\section{Article history:}

Received 30 August 2017

Received in revised form 10 November 2017

Accepted 12 November 2017

Available online 15 November 2017

Communicated by L. Ghivelder

\section{Keywords:}

Ginzburg-Landau

deGennes parameter

Mesoscopics

\begin{abstract}
A B S T R A C T
We studied the resistive state of a mesoscopic superconducting strip (bridge) at zero external applied magnetic field under a transport electric current, $J_{a}$, subjected to different types of boundary conditions. The current is applied through a metallic contact (electrode) and the boundary conditions are simulated via the deGennes extrapolation length $b$. It will be shown that the characteristic current-voltage curve follows a scaling law for different values of $b$. We also show that the value of $J_{a}$ at which the first vortexantivortex $(V-A v)$ pair penetrates the sample, as well as their average velocities and dynamics, strongly depend on the $b$ values. Our investigation was carried out by solving the two-dimensional generalized time dependent Ginzburg-Landau (GTDGL) equation.
\end{abstract}

(C) 2017 Elsevier B.V. All rights reserved.

\section{Introduction}

One mechanism for the resistive state of current carrying superconductors is the formation of hot spots, where the local temperature $T$ exceeds the transition temperature, $T_{c}$, and the superconducting order parameter is completely suppressed. It is believed that the hot spot mechanism dominates at low temperatures [1]. Another mechanism for the resistive state could be explained by the so called kinematic vortices (propagating waves of the order parameter). ${ }^{1}$ These vortices have been discovered in theoretical works [2-4] and experimentally observed by using techniques such as laser imaging [5], multiprobe voltage measurements [6], and radio-frequency synchronization $[7,8]$. They move with velocity $v_{k v} \simeq 10^{5} \mathrm{~m} / \mathrm{s}$, which is much larger than the maximal measured speed of Shubnikov-Abrikosov vortices $v_{a v} \simeq 10^{3} \mathrm{~m} / \mathrm{s}$ [5]. Because of their very high velocity, kinematic vortices do not retain their circular structure [2,3]. Berdiyorov et al. studied the dynamics of the superconducting condensate and the effect of pinning on the time response of bridges under an external applied $d c$ current.

\footnotetext{
* Corresponding author.

E-mail address: jjbarbao@unal.edu.co (J. Barba-Ortega).

1 It is already well accepted that the kinematic vortices are constituted of a $V-A v$ pair which moves at a much larger velocity than a normal Abrikosov vortex. The order parameter nearly vanishes along the line where the kinematic vortices move, although it has two minima which carry the singularities of its phase.
}

They show that, depending on the applied current, the resistive state is characterized by either the flux-flow, the phase-slip or the hot-spot states and observed qualitative changes in the dynamics of the superconducting condensate [4], [9]. In reference [10] it was shown that the $V-A v$ pairs can also be magnetically activated by applying an external magnetic field to a square mesoscopic superconductor with a concentric square hole. Very recently, this system was studied in more details by taking into account heat diffusion effects in the annihilation of the $V-A v$ pairs [11]. It was shown that the local increase of temperature should be experimentally measured by using nanoSQUIDs [12]. Fink et al. studied the effect of deGennes boundary parameter, $b$, on $T_{c}$ for various sample geometries. They found that $b$ can be used to describe a reduction or an enhancement of $T_{C}$ in small superconductors [13].

In recent works, the effect of the deGennes extrapolation length $b$ on the superconducting state of two and three dimensional specimens were studied. The structural and magnetic properties of the vortex state are significantly modified as the size of the sample is comparable to the coherence length $\xi$ and/or the London penetration depth $\lambda$ [14-16].

In this contribution, we study the resistive response of the superconducting condensate of bridges under an external applied current at zero applied magnetic field (see Fig. 1). Both sides of the bridge are attached to two electrodes symmetrically positioned (indicated by the blue color). The superconductor is covered by a very thin layer of a different material, which was adjusted by 


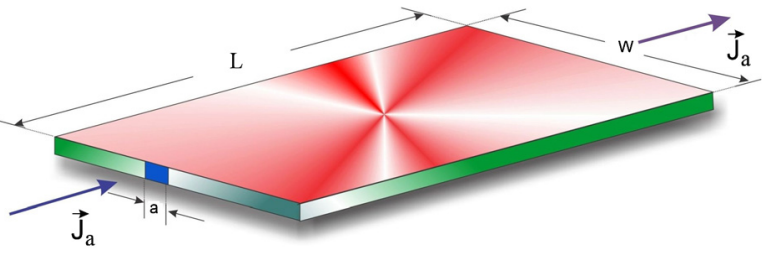

Fig. 1. (Color online) Schematic view of the studied system: a bridge of length $L$ and width $W$; the width of the electrodes is $a$, through which a uniform $d c$ current density $J_{a}$ is injected.

changing the values of $b$ (indicated by green color). A systematic study was carried out by considering different interfaces such as superconductor-vacuum ( $S C-V)$, superconductor-normal metal $(S C-M)$, superconductor-superconductor at a higher $T_{C}(S C-S C)$. We found that the value of the critical current density $J_{c 1}$, at which the first kinematic vortex enters the sample, strongly depends on the boundary conditions.

This paper is outlined as follows. In Section 2 we describe the theoretical formalism used to study a mesoscopic bridge in the presence of an applied current at zero magnetic field. Then, in Section 3 we present the results that come out from the numerical solution of the GTDGL equation for the three types of boundary conditions specified previously. In Section 4 we present our conclusions.

\section{Theoretical formalism}

In the present investigation we consider a very thin bridge of thickness $d \ll \xi$, and intermediate width $(\xi \ll W \ll \lambda)$. Within this approximation we can neglect the magnetic field produced by the transport current itself. Therefore, this can be treated as a two dimensional problem [2]. The general form of the GTDGL equation in dimensionless units is given by [17-19]:

$$
\begin{aligned}
\frac{u}{\sqrt{1+\Gamma^{2}|\psi|^{2}}} & {\left[\frac{\partial}{\partial t}+i \varphi+\frac{\Gamma^{2}}{2} \frac{\partial|\psi|^{2}}{\partial t}\right] \psi=} \\
& (\nabla-i \mathbf{A})^{2} \psi+\left(1-|\psi|^{2}\right) \psi,
\end{aligned}
$$

which is coupled to the equation for the electrostatic potential $\Delta \varphi=\operatorname{div}\{\operatorname{Im}[\bar{\psi}(\nabla-\mathrm{i} \mathbf{A}) \psi]\}$.

Here, distances are scaled by the coherence length $\xi$, time is in units of the Ginzburg-Landau time $t_{G L}=\pi \hbar / 8 k_{B} T_{c} u$, the electrostatic potential $\varphi$ is given in units of $\varphi_{0}=\hbar / 2 e t_{G L}$, the vector potential $\mathbf{A}$ is scaled by $H_{c 2} \xi$, where $H_{c 2}$ is the bulk upper critical field. From first principles, we obtain the parameters $u=5.79$ and $\Gamma=t_{E} \psi_{0} / \hbar$ (which is material dependent, $t_{E}$ being the inelastic scattering time) [17]. Neumann boundary conditions are taken at all sample boundaries, except at the electrodes where we used $\psi=0$ and $\left.\nabla \varphi\right|_{n}=-J_{a}$, where $J_{a}$ is the external applied current density in units of $J_{0}=c \sigma \hbar / 2 e t_{G L} ; \sigma$ is normal electrical conductivity. As we have mentioned above, in Eq. (1) the screening of the magnetic field is neglected, since we restrict ourselves to thin superconducting samples (see references [2], [4] and [16]). The phase diagram of mesoscopic superconductors is strongly influenced by the boundary conditions for the order parameter. In general, they are given by the deGennes boundary conditions:

$\mathbf{n} \cdot(i \nabla+\mathbf{A}) \psi=-\frac{i}{b} \psi$,

where $\mathbf{n}$ is the unit vector normal outward the superconductormedium interface, and $b$ is the deGennes surface extrapolation length. We must emphasize that the superconductor is covered by a very thin layer of another material; $b$ depends on the properties of the interface. It is maximum for an ideal surface with the mirror reflection of quasi-particles and minimum for the rough surface with the diffusive reflection [20-23]. We unify all the boundary conditions upon introducing the parameter

$\gamma= \begin{cases}1-\frac{\delta}{b}, & \text { if } b \neq 0, \\ 0, & \text { if } b=0,\end{cases}$

where $\delta$ is the resolution of the meshgrid used to solve Eq. $1 \mathrm{nu}-$ merically. For convenience, this notation allows us to obtain a more comprehensive analysis of the results. Thus (i) and $\gamma=0$ simulates an interface at the normal state, i.e., the Dirichlet boundary condition $(b=0$, where $\psi=0$ ); (ii) $0<\gamma<1$ simulates a $S C-M$ interface $(b>\delta)$; (iii) $\gamma=1$ simulates a $S C-V$ interface $(b \rightarrow \infty)$; (iv) a $S C-S C$ interface is described by $\gamma>1(b<0)$.

The first branch of the $\gamma$ parameter of Eq. (3) arises from the discretization of the deGennes boundary condition of Eq. (2) (for more details, see reference [14]); $\gamma$ relates the value of $\psi$ at the boundary with its value in an adjacent point inside the superconductor. The second branch is just a definition. Thus, we unify all the boundary conditions under consideration, $b<0, b=0, b>0$ and $b \rightarrow \infty$, in a single parameter.

Then, except at the electrodes, we employ the deGennes boundary conditions with $b \neq 0$ for the order parameter. In order to solve Eq. (1) numerically, we used the link-variable method as sketched in references [24-26]. In the numerical approximations, the $\Gamma$ parameter is the relevant one to solve Eq. (1) (see reference [14]). If $\Gamma=0$ (gapless superconductor), then there will be no kinematic vortex. In this case, the system goes straight to the normal state above a certain critical value of the $d c$ current.

\section{Results and discussion}

\subsection{The I-V characteristic curve}

We consider a bridge of width $W=8 \xi$ and length $L=12 \xi$ in absence of an external magnetic field and in the presence of a $d c$ current density $J_{a}$ uniformly applied through the electrodes of width $a=2 \xi$; we used $\Gamma=10$. The rage $10 \leq \Gamma \leq 20$ is suitable for most metals like $\mathrm{Nb}[9,17,18]$. We considered a uniform meshgrid with a resolution of 10 points per $\xi$, that is, $\delta=0.1 \xi$.

In order to analyze the response of the superconductor to an external $d c$ current, we calculated the $I-V$ characteristic curves of the bridge, which are shown in Fig. 2 for six values of the $\gamma$ parameter. As we can see from this figure, the values of $J_{c 1}$ for which a resistive state takes place are $1.08,1.22,1.36,1.74,2.12,2.60$ for $\gamma=0,0.8,0.9,1,1.05,1.1$ respectively. The small resistance for $J_{a}<J_{c 1}$ is due solely to the electrodes. Note that for $\gamma<1$ $(\gamma>1) J_{c 1}$ decreases (increases) compared to the $S C-V$ interface critical current density. The striking result is the extent for which the resistive phase persists before the system goes to the normal state for a small increase of $\gamma$. In addition, we can observe that a small decrease in the value of $\gamma$ substantially diminishes the value of the critical current density. This is an important result, since in the fabrication process of superconducting samples, it is inevitable the contamination of the material at the borders, which produces a value of $\gamma<1$.

For $J_{a}>J_{c 1}$, the system goes into a resistive state with a finite jump in the output voltage, signaled by a discontinuity in the resistance $\partial V / \partial J_{a}$ as a function of the applied current $J_{a}$. The results are shown in Fig. 3 for the boundary conditions $\gamma=0.8,1,1.05$. This resistive state is characterized by the fast-moving vortices as reported in several works $[27,28]$. The dynamics of the $V-A v$ pairs will be discussed in more details in subsection 3.3.

\subsection{The first critical current density}

Now we will determine the relationship between $J_{c 1}$ and $\gamma$. For this end, we registered the values of the first critical current density for several boundary conditions and in Fig. 4 it is plotted $J_{c 1}$ 


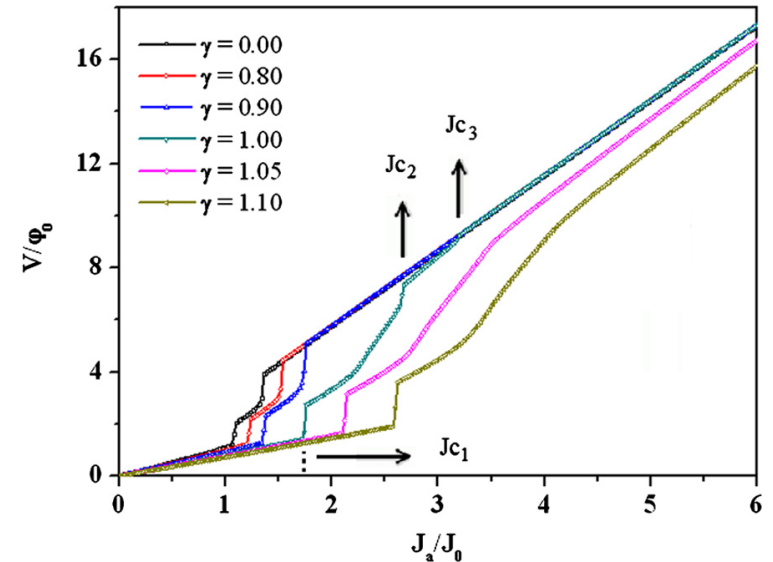

Fig. 2. Time-averaged voltage $V$ as a function of the applied current-density $J_{a}$ for several values of $\gamma$. The arrows indicate the critical current densities only for the $S C-V$ interface $(\gamma=1)$.

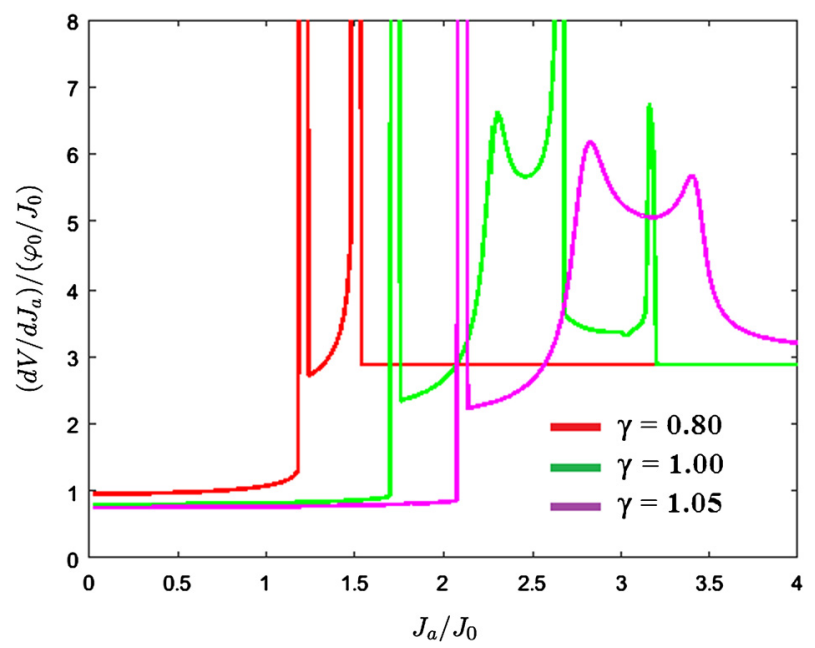

Fig. 3. (Color online) Curve of the resistivity $\partial V / \partial J_{a}$ as a function of the applied current $J_{a}$ for the indicated boundary conditions.

as a function of $\gamma$. As we can see, $J_{c 1}$ increases exponentially with $\gamma$, according to the expression $J_{c 1} \simeq 1.080+0.0002 \exp (\gamma / 0.123)$ (blue line in Fig. 4). The rapid growth of $J_{c 1}$ means that the smaller the metallic boundary is, i.e., the more enhanced the surface superconductivity is, the more diamagnetic the material will be. From an experimental point of view, this is an important result, since the appearance of a resistive state occurs for larger $J_{c 1}$ as $\gamma$ increases. Thus, the shielding of the first penetration of the kinematic vortices could be strongly enhanced by surrounding the bridge by a thin layer of another superconducting material of higher $T_{c}$.

\subsection{The $V-A v$ nucleation dynamics}

Now, we will turn our discussion to the dynamics of the nucleation of the $V-A v$ pairs. But first, let us make some considerations of results previously found. In the pioneering work of Andronov et al. [2], it is was found that, if $a=W$, the current distribution is uniform in the longitudinal direction. In this case, the order parameter is uniform along the channel $(x=L / 2 \text { line })^{2}$ Thus, no instability is produced in the resistive phase and the system goes

\footnotetext{
2 It should be noted that in this work we use the term channel to denominate the line through which the kinematic vortices will move. In reference [2], it was used to denominate the superconducting strip itself.
}

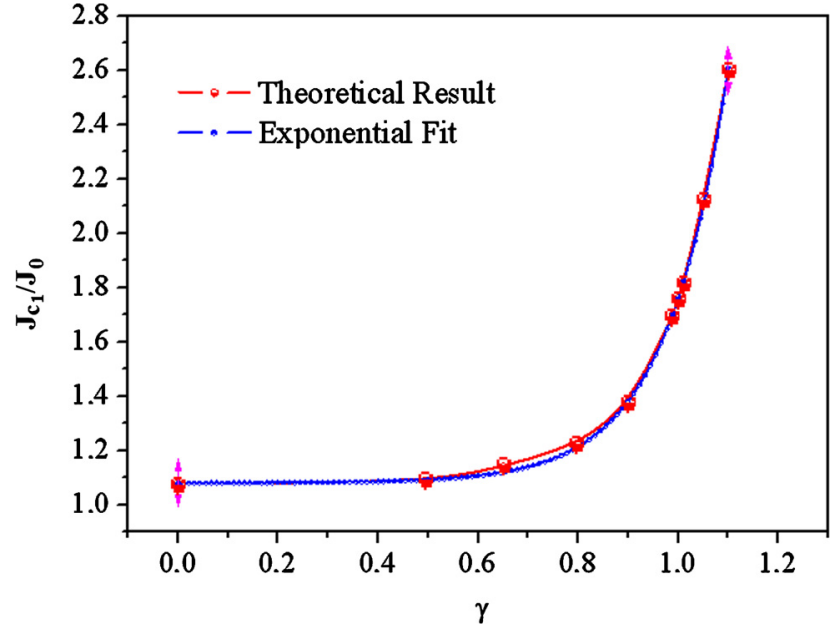

Fig. 4. (Color online) $J_{c 1}$ as a function of $\gamma$; theoretical results (red line) and exponential fit (blue line).

straight to the normal state at $J_{a}=J_{c 1}$. However, if an inhomogeneity is introduced (say, a defect) along the channel, both the potential and the order parameter become unstable for $J_{a}>J_{c 1}$. In this scenario, the kinematic vortices constituted by a $V-A v$ pair start nucleating in the sample. In reference [3] it was argued that the inhomogeneity across the transversal direction is sufficient to originate the kinematic vortices, but not necessary. If we consider an uniform channel, but take an electrode smaller then the width of the bridge, a locally injected current density will produce an electronic instability in the superconducting condensate along the transversal channel.

The authors of reference [3] have argued that, for $J_{a}>J_{c 1}$ the kinematic vortices first sprout at the center of the channel and propagate towards the edges and finally disappear. When $J_{a}$ achieves a determined value, there is an inversion in the formation of the $V-A v$ pairs, they nucleate at the edges and move towards the center where they annihilate one each other. This inversion on the nucleation fairly coincides with the maximum of the resistance in the interval $J_{c 1}<J_{a}<J_{c 2}$ (see Fig. 3). The nucleation and annihilation of the $V-A v$ pairs persist until $J_{a}=J_{c 2}$. From Fig. 3, we can see that for $\gamma=1$ we have obtained exactly the same result as the equivalent one found in reference [3]. In the interval $J_{c 2}<J_{a}<J_{c 3}$ the system is in a partially normal state where the superconductivity is nearly destroyed in the whole bridge, except at the corners.

In the context of references [2] and [3], we have two factors responsible for the vortex dynamics in the resistive state, either a defect is present along the channel or the electrode size is smaller then the width of the bridge, $a<W$. Here, we have an additional factor which is the deGennes extrapolation length. We have observed that whether the local inversion on the formation of the $V-A v$ pairs occurs or not also depends on the values of $\gamma$.

In Fig. 5 it is plotted the average velocity, $V_{m}$, of a vortex during the annihilation with an antivortex. The upper panel is for the $S C-M$ interfaces simulated with $\gamma=0.6,0.8,0.9$, and in the lower panel it is shown the $V_{m}$ curves for the SC-SC interfaces simulated with $\gamma=1.05,1.1$. In this last case, we can see that, as $\gamma$ increases, the intensity of the peak of $V_{m}$ decreases and is shifted towards larger values of $J_{a}$. For $0<\gamma<1, V_{m}$ decreases monotonically as $J_{a}$ increases. Such samples present only one type of $V-A v$ dynamics, i.e., all the pairs are formed at the center perpendicular to the current direction and annihilate at the border of the sample (see Fig. 6). This behavior is a coherent physical result, since for metal interfaces, the degradation of the superconductivity on the edge of the bridge precipitates the nucleation of the normal 

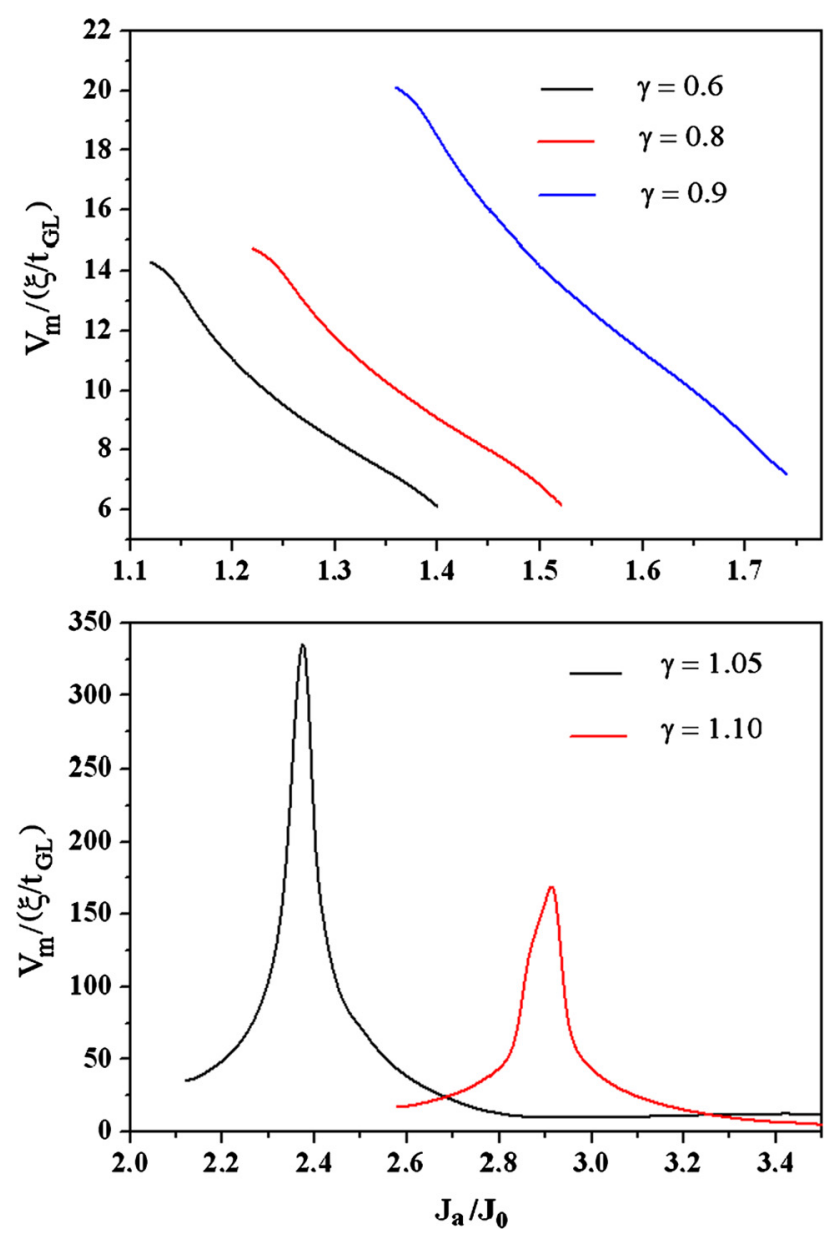

Fig. 5. (Color online) Average velocity of the $V-A v$ pairs, $V_{m}$, for a $S C-M$ interfaces simulated with $0<\gamma<1$ (upper panel) and for the $S C$-SC at higher critical temperature interfaces simulated with $\gamma>1$ (lower panel).

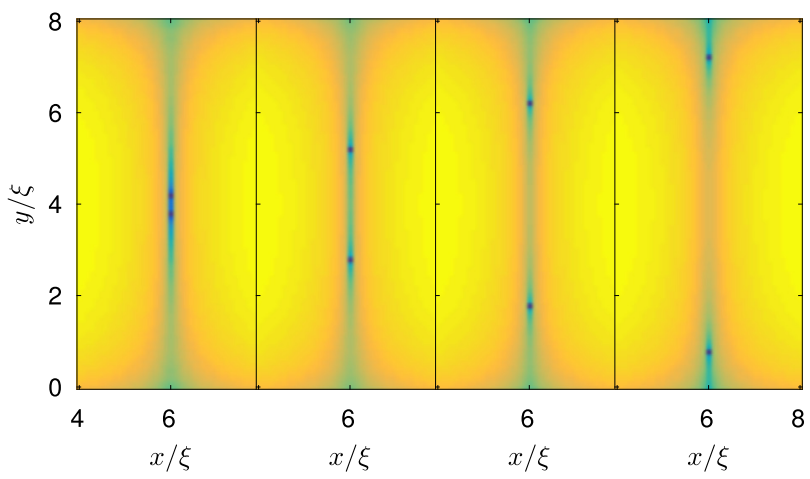

Fig. 6. (Color online) Snapshots of the logarithm of the order parameter, $\ln |\psi|$, (yellow/blue corresponds to largest/zero $|\psi|$ ) for $\gamma=0.8$ and $J_{a}=1.38 J_{0}$. In this case the $V-A v$ pairs always nucleate at the center of the sample and are ejected through the edges.

state and there is no time for an inflection in the $I-V$ characteristic curve between the two transitions, as can be seen from Fig. 3 .

For $\gamma>1$, the dynamics is similar to the $S C-V$ case. First, the $V-A v$ pairs are nucleated at the center of the sample and then move along the channel until they are ejected at the borders of the bridge, as illustrated in Fig. 7. After the maximum of the average velocity (see lower panel of Fig. 5) the kinematic vortices are formed at the edge of the bridge and annihilate one each other at the center, as can be seen from Fig. 8.

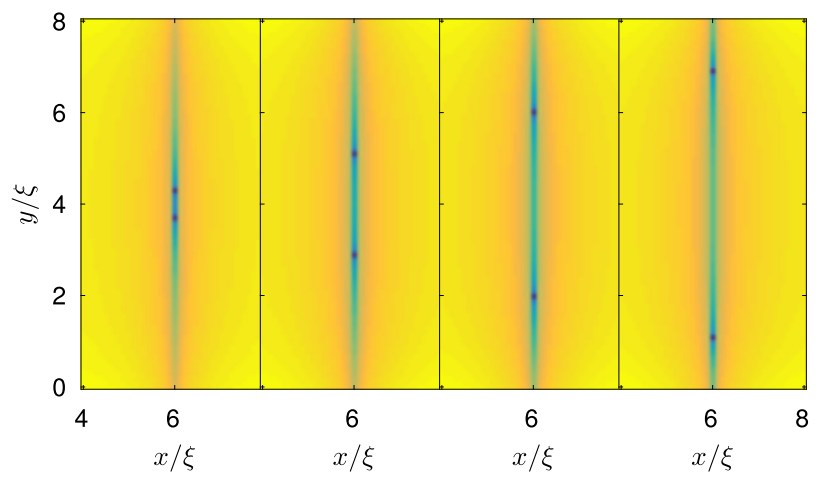

Fig. 7. (Color online) The same as Fig. 6 for $\gamma=1.05$ and $J_{a}=2.22 J_{0}$. In this case, for $J_{c 1} \leq J_{a} \leq 2.80 J_{0}$, the $V-A v$ pairs always nucleate at the center of the sample and are ejected at the edges.

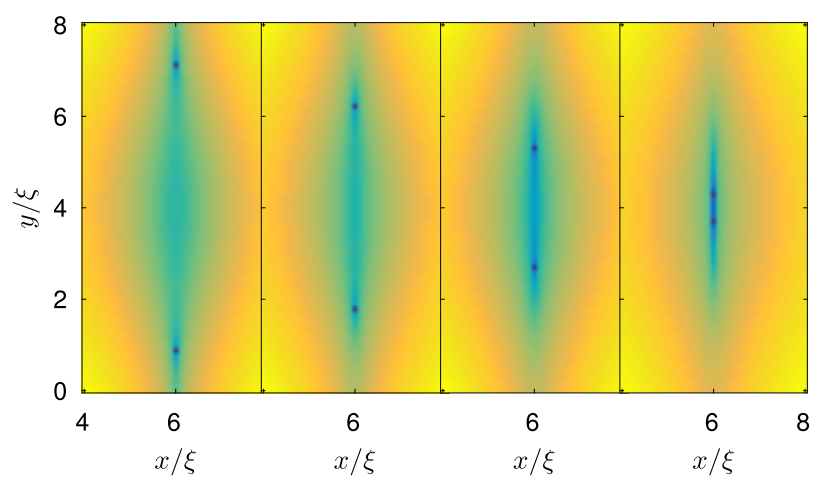

Fig. 8. (Color online) The same as Fig. 7 for $\gamma=1.05$ and $J_{a}=3.12 J_{0}$. In this situation, for $J_{a}>2.80 J_{0}$, there is an inversion in the formation the $V-A v$ pairs. They always nucleate at the border of the sample and are annihilated at the center.

As a final remark, we note that the order of magnitude of the average velocity of the $V-A v$ pairs are much lower for the $S C$ $S C$ interface than for the $S C-V$ counterpart. This can be explained as follows. For $\gamma>1$, superconductivity is considerably enhanced throughout the bridge. Thus, the medium becomes more viscous for the $V-A v$ pairs. This is an important result for controlling the speed of these objects, so that we can avoid heating generation.

\section{Conclusions}

By solving the GTDGL equation, we studied the resistive state of superconducting bridges under an applied $d c$ electrical current for different boundary conditions. We found that the critical current for the transition to the resistive state, $J_{c 1}$, shows a strong dependence with the type of boundary conditions. We have found that, a superconductor-superconductor at a higher $T_{c}$ boundary avoids the appearance of resistive state, increasing $J_{c 1}$. We also found an analytical dependence of $J_{c 1}$ with the $\gamma$ parameter, and have shown that the dynamics of the $V-A v$ along the central channel of the bridge strongly depends on this parameter. Thus, our results are of experimental importance since the kinematic vortex dynamics can be controlled by depositing other materials at the boundaries of mesoscopic superconducting devices.

\section{Acknowledgements}

We thank the Brazilian Agencies CNPq and the São Paulo Research Foundation (FAPESP), grants 2012/04388-0 and 2016/ 12390-6, for financial support. 


\section{References}

[1] W.J. Skocpol, M.R. Beasley, M. Tinkham, J. Appl. Phys. 45 (1974) 4054.

[2] A. Andronov, I. Gordion, V. Kurin, I. Nefedov, I. Shereshevsky, Physica C 213 (1993) 193.

[3] G. Berdiyorov, M.V. Milošević, F.M. Peeters, Phys. Rev. B 79 (2009) 184506.

[4] G. Berdiyorov, K. Harrabi, F. Oktasendra, K. Gasmi, A.I. Mansour, J.P. Maneval, F.M. Peeters, Phys. Rev. B 90 (2014) 054506.

[5] A.G. Sivakov, A.M. Glukhov, A.N. Omelyanchouk, Y. Koval, P. Muller, A.V. Ustinov, Phys. Rev. Lett. 91 (2003) 267001.

[6] G.J. Dolan, L.D. Jackel, Phys. Rev. Lett. 39 (1977) 1628.

[7] R.B. Laibowitz, A.N. Broers, J.T.C. Yeh, J.M. Viggiano, Appl. Phys. Lett. 35 (1979) 891.

[8] W.J. Skocpol, M.R. Beasley, M. Tinkham, J. Low Temp. Phys. 16 (1974) 145.

[9] G. Berdiyorov, K. Harrabi, J.P. Maneval, F.M. Peeters, Supercond. Sci. Technol. 28 (2015) 025004.

[10] E. Sardella, P.N. Lisboa-Filho, C.C.S. Silva, L.R.E. Cabral, W.A. Ortiz, Phys. Rev. B 80 (2009) 012506.

[11] E.C.S. Duarte, E. Sardella, W.A. Ortiz, R. Zadorosny, J. Phys. Condens. Matter 29 (2017) 405605.

[12] D. Halbertal, J. Cuppens, M. Ben Shalom, L. Embon, N. Shadmi, Y. Anahory, H.R. Naren, J. Sarkar, A. Uri, Y. Ronen, Y. Myasoedov, L.S. Levitov, E. Joselevich, A.K. Geim, E. Zeldov, Nature 539 (2016) 470.

[13] H. Fink, S. Haley, C.V. Giuraniucz, V.F. Kozhevnikovz, J.O. Indekeu, Mol. Phys. 103 (21) (2005) 2969.
[14] J. Barba-Ortega, E. Sardella, J.A. Aguiar, Supercond. Sci. Technol. 24 (2011) 015001.

[15] J. Barba-Ortega, E. Sardella, J.A. Aguiar, Phys. Lett. A 379 (2015) 732.

[16] M. Tinkham, J. Low Temp. Phys. 35 (1979) 147.

[17] L. Kramer, R.J. Watts-Tobin, Phys. Rev. Lett. 40 (1978) 1041.

[18] J. Watts-Tobin, Y. Krähenbühl, L. Kramer, J. Low Temp. Phys. 42 (1981) 459.

[19] D.Y. Vodolazov, F.M. Peeters, M. Morelle, V.V. Moshchalkov, Phys. Rev. B 71 (2005) 184502.

[20] P.G. de Gennes, Superconductivity of Metals and Alloys, Addison-Wesley, New York, 1994.

[21] P.G. de Gennes, J. Matricon, Rev. Mod. Phys. 36 (1964) 45.

[22] E.A. Andrushin, V.L. Ginzburg, A.P. Silin, Usp. Fiz. Nauk 163 (1997) 105.

[23] R.O. Zaitsev, Zh. Eksp. Teor. Fiz. 48 (1965) 1759.

[24] W.D. Gropp, H.G. Kaper, G.K. Leaf, D.M. Levine, M. Palumbo, V.M. Vinokur, J. Comput. Phys. 123 (1996) 254.

[25] G. Buscaglia, C. Bolech, C. Lopez, in: J. Berger, J. Rubinstein (Eds.), Connectivity and Superconductivity, Springer, Heidelberg, 2000.

[26] M.V. Milošević, R. Geurts, Physica C 470 (2010) 791.

[27] A.V. Silhanek, M.V. Milošević, R.B.G. Kramer, G.R. Berdiyorov, J. Van de Vondel, R.F. Luccas, T. Puig, F.M. Peeters, V.V. Moshchalkov, Phys. Rev. Lett. 104 (2010) 017001.

[28] L.N. Bulaevskii, M.J. Graf, V.G. Kogan, Phys. Rev. B 85 (2012) 014505. 